\title{
(C) OPEN ACCESS \\ The implementation of cardiac arrest treatment recommendations in English acute NHS trusts: a national survey
}

\author{
James Carberry, ${ }^{1}$ Keith Couper, ${ }^{2,3}$ Joyce Yeung ${ }^{1,3}$
}

\begin{abstract}
${ }^{1}$ University of Birmingham, Edgbaston, Birmingham, UK ${ }^{2}$ Warwick Medical School, University of Warwick, Coventry, UK

${ }^{3}$ Academic Department of Anaesthesia, Critical Care, Pain and Resuscitation, Heart of England NHS Foundation Trust, Birmingham, UK
\end{abstract}

\section{Correspondence to}

Dr Joyce Yeung, Institute of Inflammation and Ageing, College of Medical and Dental Sciences, University of Birmingham, Edgbaston, Birmingham, B15 2TT, UK; j.yeung@bham.ac.uk

Received 8 December 2016 Revised 23 March 2017

Accepted 2 April 2017 Published Online First 25 April 2017
ABSTRACT

Purpose of the study There are approximately 35000 in-hospital cardiac arrests in the UK each year. Successful resuscitation requires integration of the medical science, training and education of clinicians and implementation of best practice in the clinical setting. In 2015, the International Liaison Committee on Resuscitation (ILCOR) published its latest resuscitation treatment commendations. It is currently unknown the extent to which these treatment recommendations have been successfully implemented in practice in English NHS acute hospital trusts.

Methods We conducted an electronic survey of English acute NHS trusts to assess the implementation of key ILCOR resuscitation treatment recommendations in relation to in-hospital cardiac arrest practice at English NHS acute hospital trusts.

Results of 137 eligible trusts, 73 responded to the survey (response rate 53.3\%). The survey identified significant variation in the implementation of ILCOR recommendations. In particular, the use of waveform capnography $(n=33,45.2 \%)$ and ultrasound $(n=29$, $39.7 \%$ ) was often reported to be available only in specialist areas. Post-resuscitation debriefing occurs following every in-hospital cardiac arrest in few trusts $(5.5 \%, n=4)$, despite a strong ILCOR recommendation. In contrast, participation in a range of quality improvement strategies such as the National Cardiac Arrest Audit $(90.4 \%, n=66)$ and resuscitation equipment provision/ audit $(91.8 \%, n=67)$ were high. Financial restrictions were identified by $65.8 \%(n=48)$ as the main barrier to guideline implementation.

Conclusion Our survey found that ILCOR treatment recommendations had not been fully implemented in most English NHS acute hospital trusts. Further work is required to better understand barriers to implementation.

\section{INTRODUCTION}

In-hospital cardiac arrest (IHCA) is a true medical emergency, in which the delivery of time-critical high-quality care is challenging. In the UK there are 1.5 IHCAs per 1000 hospital admissions, with just $18.4 \%$ of patients surviving to hospital discharge. ${ }^{1}$

In the UK, cardiac arrest treatment guidelines are developed by the Resuscitation Council (UK). ${ }^{2}$ CrossMark These guidelines are based on treatment recommendations developed by the International Liaison Committee on Resuscitation (ILCOR) which are reviewed and published every 5 years in the journals Circulation and Resuscitation. ${ }^{2-4}$ The latest ILCOR review process, which concluded in 2015, involved the completion of 169 systematic reviews across seven domains (adult basic life support; adult advanced life support; acute coronary syndrome; paediatric basic life support; neonatal resuscitation; education, implementation and teams; first aid). ${ }^{3}$ These reviews were conducted using the robust GRADE (Grading of Recommendations Assessment, Development and Evaluation) methodology, which culminated in teams of resuscitation experts making a recommendation or suggestion for or against an intervention. ${ }^{56}$

The Resuscitation Council (UK) guidelines and the ILCOR treatment recommendations on science are broadly similar, although there are subtle differences due to the purpose of the two documents. $^{2}{ }^{3}$ The key difference is that the ILCOR treatment recommendations are broader in scope and cover all elements of resuscitation science. This includes detailed recommendations on acute coronary syndrome and training delivery, which are not covered in detail in the Resuscitation Council (UK) guidelines. This approach ensures that the Resuscitation Council (UK) guidelines are tailored to the UK context.

The cardiac arrest formula for survival describes three components that are essential to optimise survival following cardiac arrest-namely, medical science, education and implementation. ${ }^{7}$ This concept of a formula describes survival as a function of how well each of the three components exist in practice. As such, for optimal survival, medical science must be developed, taught and implemented in practice. However, it is presently unknown how well and quickly resuscitation treatment recommendations are implemented by English acute hospital trusts. The publication of the latest ILCOR treatment recommendations in October 2015 provides a timely opportunity to review this implementation. ${ }^{3}$

\section{METHODS}

Between February and March 2016 we conducted a national electronic survey of all English acute NHS trusts to assess their implementation of the 2015 ILCOR treatment recommendations. Baseline data on key NHS trust characteristics, such as the number of hospitals and beds, were collected from Department of Health data. The electronic survey was hosted by SurveyMonkey (Palo Alto, California, USA).

The main questionnaire comprised 28 questions that covered the implementation of key treatment recommendations across four ILCOR domains: 
adult basic life support (BLS); adult advanced life support (ALS); acute coronary syndrome (ACS); and education, implementation and teams (EIT). ${ }^{8-11}$ To develop the survey, the study authors reviewed the 2015 ILCOR treatment recommendations and developed a pool of questions relevant to IHCA practice. The authors then ranked specific topic areas based on their perceived importance in terms of relevance to UK hospital setting and uncertainty of practice. As many questions considered important were selected as possible, while attempting to ensure that the survey was simple to navigate and quick to complete in order to maximise the response rate. The survey was piloted with resuscitation officers from two NHS trusts to ensure that the questions were valid and would be interpreted correctly by the intended audience. Minor amendments to the survey were made following feedback.

\section{Inclusion/exclusion criteria}

NHS trusts in England were eligible to participate in the survey if they were an NHS hospital trust that provided general acute adult secondary care. NHS trusts were not eligible if they were a specialist trust providing only paediatric care, women's care, mental healthcare or tertiary services.

\section{Data collection}

Eligible trusts were identified using a Department of Health list of NHS trusts. ${ }^{12}$ Resuscitation officers are specialist practitioners that typically have a combined training, clinical service, quality assurance and research role in the area of resuscitation. ${ }^{13}$ As such, trust resuscitation officers were identified as the most appropriate person to complete the survey on behalf of their respective NHS trust.

A three-stage contact approach was adopted. First, an initial email was sent to the resuscitation officer at each eligible trust inviting them to complete the survey on behalf of the trust. A second reminder email was sent a week later if there was no reply. Finally, in the event of further non-response, the resuscitation officer was contacted by telephone.

\section{Data analysis}

Data were downloaded and imported into Microsoft Excel (Microsoft Corporation, Washington, USA) to facilitate a descriptive analysis. Categorical data are reported as frequency and percentage. Continuous data were assessed for normality. Normally distributed continuous data are reported as mean and 95\% CI. Non-normally distributed data are reported as median and IQR.

\section{RESULTS}

A total of 157 acute NHS trusts were identified in England, of which 20 were deemed not eligible to participate in the study due to being a mental health trust $(n=2)$, paediatric care only trust $(n=4)$, women's care only trust $(n=2)$ or a trust providing only specialist tertiary services $(n=12)$ (figure 1$)$. Of the 137 eligible trusts, the survey was completed by 73 trusts (response rate $53.3 \%$ ).

\section{Characteristics of participating hospitals}

Most responding trusts managed more than one hospital $(n=45$, $61.9 \%$ ), with a median number of hospital beds of 660 (IQR 450-950). The median numbers of whole time equivalent (WTE) junior and senior resuscitation officers funded by each trust was 1 (IQR 0-2.1) and 2 (IQR 1-3), respectively. Most trusts $(\mathrm{n}=32$, $43.8 \%)$ reported a cardiac arrest incidence of 1-1.99 IHCAs per 1000 admissions, with 16 (21.9\%) respondents unsure of the IHCA incidence at their trusts. There were small differences

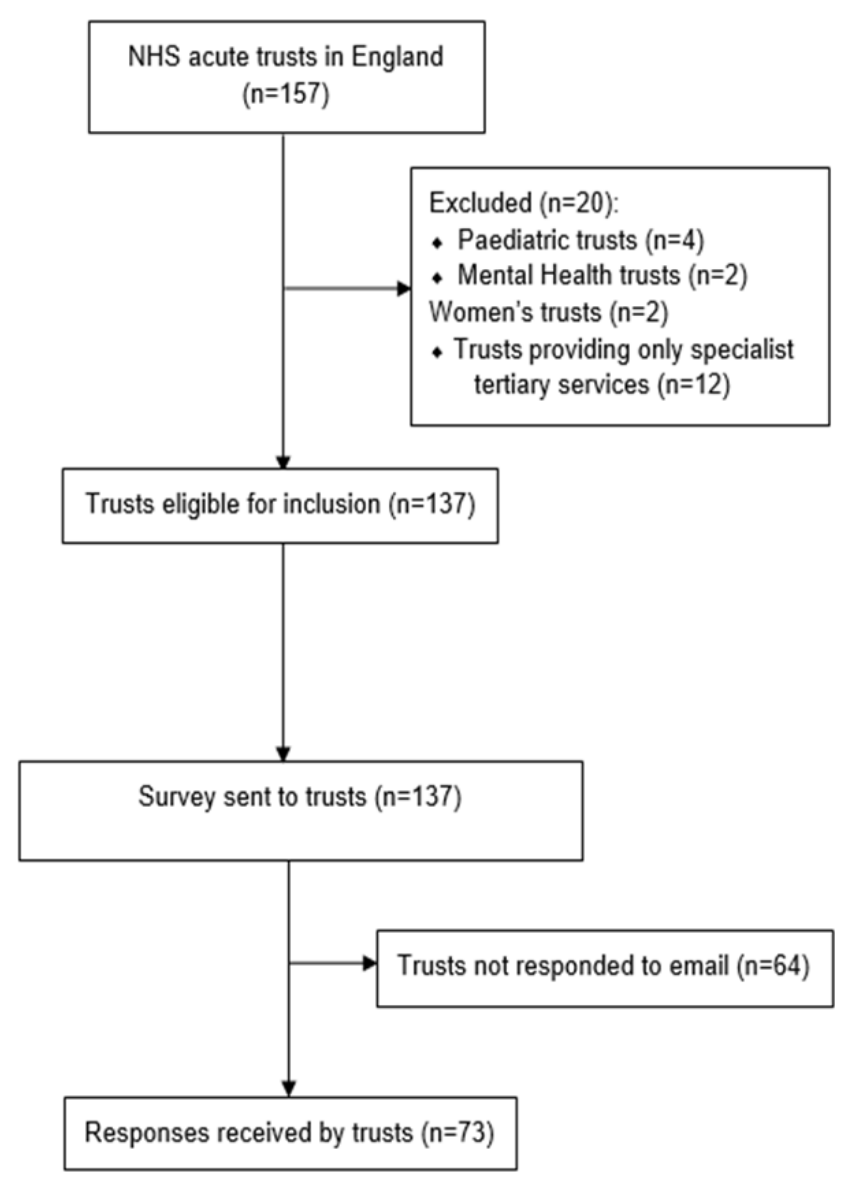

Figure 1 Progress through the study from identification of target population to completion of survey ( $n=$ number of trusts).

between responding and non-responding trusts in relation to the number of hospitals managed and number of beds (table 1 ).

Tables 2-4 summarise the implementation of guidelines in relation to devices and treatments; pre-briefing and

\begin{tabular}{lll}
\hline $\begin{array}{l}\text { Table } 1 \\
\text { hospitals }\end{array}$ & Characteristics of participating and non-participating \\
\hline & $\begin{array}{l}\text { Responding } \\
\text { hospitals ( } \mathbf{n}=73)\end{array}$ & $\begin{array}{l}\text { Non-responding } \\
\text { hospitals ( } \mathbf{n}=64)\end{array}$ \\
\hline Hospitals managed by trust, $\mathrm{n}(\%)$ & $28(38.4)$ & $17(26.6)$ \\
\hline 1 & $22(30.1)$ & $18(28.1)$ \\
\hline 2 & $12(16.4)$ & $13(20.3)$ \\
\hline 3 & $11(15.1)$ & $16(25.0)$ \\
$\geq 4$ & $660(450-950)$ & $769(580-1027)$ \\
\hline Number of beds, median (IQR) & & \\
$\begin{array}{l}\text { Number of WTE resuscitation officers, } \\
\text { median (IQR) }\end{array}$ & $1(0-2.1)$ & \\
\hline Junior (band 5/6) & $2(1-3)$ & \\
\hline Senior (band 7/8) & & \\
\hline Cardiac arrests per 1000 admissions, & & \\
$\mathrm{n}$ (\%) & $7(9.6)$ & \\
\hline $0-0.99$ & $32(43.8)$ & \\
\hline $1-1.99$ & $10(13.7)$ & $8(11.0)$ \\
\hline $2-2.99$ & $16(21.9)$ & \\
$\geq 3$ & & \\
\hline Unknown & & \\
\hline WTE, whole time equivalent. & & \\
\hline
\end{tabular}


Table 2 Devices and treatments

Respondents

$(n=73) \quad$ ILCOR recommendation 2015

Mechanical chest compression device

Hospital has mechanical device, $\mathrm{n}(\%)$

$49(67.1)$

'We suggest against the routine use of automated

Devices currently used by hospitals* $\mathrm{n}(\%)$ mechanical chest compression devices but suggest they Autopulse (ZOLL Medical Corporation, Chelmsford, MA, USA) are a reasonable alternative to use in situations where

LUCAS (Physio-Control Inc/Jolife AB, Lund, Sweden)

$19(38.8)$

35 (71.4)

Indications for use of a mechanical device ${ }^{*}, \mathrm{n}(\%)$ sustained high-quality manual chest compressions are impractical or compromise provider safety' (Weak recommendation) ${ }^{10}$

Routinely used at all cardiac arrests

ED cardiac arrests

$21(42.9)$

Cardiac catheter laboratory cardiac arrests

$26(53.1)$

Patients in cardiac arrest requiring transfer

$10(20.4)$

Cardiac arrest in CT scanner

Prolonged cardiac arrest

$32(65.3)$

Mechanical device not routinely available

Waveform gapnography

Hospital routinely uses waveform capnography during cardiac arrest events, $\mathrm{n}(\%)$

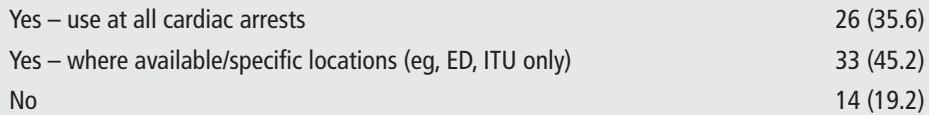

Ultrasound

Ultrasound used during CPR, $\mathrm{n}(\%)$

Yes - routinely available on all wards

Yes - if skilled personnel available

Yes - restricted to ED/ITU

No

Extracorporeal membrane oxygenation

Hospital has access to extracorporeal membrane oxygenation for cardiac arrest patients, $n(\%) \quad 8(11.0)$

CPR prompt/ feedback devices

CPR prompt/feedback devices used by hospitals during CPR, $\mathrm{n}(\%)$

Metronome

$10(13.7)$

Accelerometer-based device

$12(16.4)$

Other device

$2(2.7)$

CPR prompt/feedback devices not used routinely during CPR

$54(74.0)$

'We suggest ECPR is a reasonable rescue therapy for selected patients with cardiac arrest when initial conventional CPR is failing in settings where this can be implemented' (weak recommendation) ${ }^{10}$

'We suggest the use of real-time audiovisual feedback and prompt devices during CPR in clinical practice as part of a comprehensive system for care for cardiac arrest (weak recommendation) $^{8}$ continuously monitor the position of a tracheal tube during CPR in addition to clinical assessment' (strong recommendation $)^{10}$

\section{without interfering with standard ACLS protocol, it may considered as an additional diagnostic tool to identify potentially reversible causes' (weak recommendation) ${ }^{10}$}

'We suggest against the use of real-time audiovisual feedback and prompt devices in isolation (ie, not part of a comprehensive system of care)' (weak ${\text { recommendation })^{8}}^{8}$

Patients where primary percutaneous coronary intervention is considered post-arrest, $\mathrm{n}(\%)$

STEMI

$50(68.5)$

Other (not STEMI) with ECG changes and likely cardiac cause

$29(39.7)$

All patients with likely cardiac cause

$26(35.6)$

PCI not available - thrombolysis considered for STEMI

$1(1.4)$

No patients

$0(0)$

Unsure
'We recommend emergency cardiac catheterisation laboratory evaluation in comparison with cardiac catheterisation later in the hospital stay or no catheterisation in select adult patients with ROSC after OHCA of suspected cardiac origin with ST elevation on ECG' (strong recommendation)?

'We suggest emergency cardiac catheterisation laboratory evaluation in comparison with cardiac catheterisation later in the hospital stay or no catheterisation in select adult patients who are comatose with ROSC after OHCA of suspected cardiac origin without ST elevation on ECG' (weak recommendation) ${ }^{7}$

*Multiple answers allowed.

ILCOR, International Liaison Committee on Resuscitation; OHCA, Out of Hospital Cardiac Arrest; ROSC, Return of Spontaneous Circulation; STEMI, ST-elevation myocardial infarction.

debriefing; BLS and ALS training provision. Each table incorporates the relevant ILCOR treatment recommendation for each question. ${ }^{8-11}$

\section{Devices and treatment during cardiac arrests in the clinical setting}

Respondents were asked about the availability of medical devices that were cited by the international resuscitation guidelines (table 2). Mechanical chest compression devices that could deliver consistent high quality chest compressions were owned by $67.1 \%$ of trusts surveyed $(n=49)$, with common indications for use reported to be prolonged cardiac arrests $(n=32,65.3 \%)$, cardiac catheter laboratory cardiac arrests $(n=26,53.1 \%)$ and emergency department cardiac arrests $(n=21,42.9 \%)$. Only one trust reported that devices are used routinely at all cardiac arrests $(\mathrm{n}=1,2.0 \%)$ 


\begin{tabular}{|c|c|c|}
\hline \multicolumn{3}{|l|}{ Debriefing } \\
\hline \multicolumn{3}{|c|}{$\begin{array}{l}\text { Trust runs formal programme for staff feedback/debriefing on their performance following IHCA, } \\
\mathrm{n}(\%)\end{array}$} \\
\hline Yes - for every arrest & $4(5.5)$ & \multirow{14}{*}{$\begin{array}{l}\text { We recommend data-driven, performance- } \\
\text { focused debriefing of rescuers after } \\
\text { IHCA in both adults and children' (strong } \\
\text { recommendation) }\end{array}$} \\
\hline Yes - for some arrests & $36(49.3)$ & \\
\hline Unsure & $2(2.7)$ & \\
\hline No & $31(42.5)$ & \\
\hline \multicolumn{2}{|l|}{ Types of feedback/debriefing offered to staff, $n(\%)$} & \\
\hline Hot debrief (without CPR quality data) & $33(82.5)$ & \\
\hline Hot debrief (with CPR quality data) & $3(7.5)$ & \\
\hline Cold debrief (without CPR quality data) & $26(65.0)$ & \\
\hline Cold debrief (with CPR quality data) & $7(17.5)$ & \\
\hline Written feedback (without CPR quality data) & $5(12.5)$ & \\
\hline Written feedback (with CPR quality data) & $2(5.0)$ & \\
\hline \multicolumn{2}{|l|}{ Focus of debrief (1, not important to 5 , key focus) } & \\
\hline Education/quality of care issues, median (IQR) & $4(4-5)$ & \\
\hline Psychological/emotional issues, median (IQR) & $4(4-5)$ & \\
\hline \multicolumn{3}{|l|}{ Quality Improvement } \\
\hline \multicolumn{3}{|l|}{ Quality improvement strategies in use at hospitals, $\mathrm{n}(\%)$} \\
\hline Participation in National Cardiac Arrest Audit (NCAA) & $66(90.4)$ & \multirow{10}{*}{$\begin{array}{l}\text { 'We suggest the use of performance } \\
\text { measurement and quality improvement } \\
\text { initiatives in organisations that treat } \\
\text { cardiac arrest' (weak recommendation) }\end{array}$} \\
\hline Patient outcome review & $49(67.1)$ & \\
\hline CPR quality review & $21(28.8)$ & \\
\hline Rolling CPR refreshers & $49(67.1)$ & \\
\hline In situ cardiac arrest simulation & $52(71.2)$ & \\
\hline Real-time CPR feedback & $21(28.8)$ & \\
\hline Debriefing & $41(56.2)$ & \\
\hline DNAR documentation audit/review & $66(90.4)$ & \\
\hline Incident reporting review & $57(78.1)$ & \\
\hline Resuscitation equipment provision/audit & $67(91.8)$ & \\
\hline
\end{tabular}

DNAR, Do Not Attempt Resuscitation; ILCOR, International Liaison Committee on Resuscitation.

Ultrasound $(n=66,90.4 \%)$ and waveform capnography $(n=59$, $80.8 \%$ ) were reported to be available at the majority of trusts, but their use was not routine during cardiac arrests. In particular, waveform capnography was often available in specialist clinical areas only, with only a minority of trusts reporting its availability at all cardiac arrests $(n=26,35.6 \%)$. Similarly, ultrasound was not routinely used with only three $(4.1 \%)$ trusts reporting it routinely available at all cardiac arrests.

Most trusts $(n=54,74.0 \%)$ did not use a CPR prompt or feedback device to monitor quality of CPR during IHCAs. In trusts where a device was used, accelerometer-based devices were the most commonly used devices, which were used in 12 (16.4\%) trusts.

In terms of treatment for cardiac arrests, access to the use of extracorporeal membrane oxygenation for patients in cardiac arrest was very limited and only available in eight $(11.0 \%)$ trusts.

In most trusts $(68.5 \%, \mathrm{n}=50)$, patients with ST-elevation myocardial infarction (STEMI) were considered for primary percutaneous coronary intervention (pPCI), either at their own centre or referred for consideration elsewhere. Patients without STEMI but who had ECG changes and a likely cardiac cause of the arrest were considered for pPCI at 29 (39.7\%) trusts. There were a similar number of trusts $(n=26,35.6 \%)$ where all patients with a likely cardiac cause of the arrest were considered for pPCI.

\section{Debriefing and quality improvement}

Post cardiac arrest debriefing can be used to educate staff and improve overall CPR quality. Just over half of trusts $(n=40$,
54.7\%) reported that debriefing is provided following cardiac arrest (table 3). In trusts that provided debriefing, CPR quality data were not routinely used, with most commonly used modalities being an immediate (hot) debrief without CPR quality data $(\mathrm{n}=33,82.5 \%)$ and a delayed (cold) debrief without CPR quality data $(n=26,65.0 \%)$. It is possible that CPR quality data were simply not available due to the lack of feedback devices used in cardiac arrests. Both educational (quality of care) and psychological (emotional) issues were considered important focuses of debriefing by trusts.

The vast majority of responding trusts were engaged in quality improvement interventions with more than 90\% participating in the National Cardiac Arrest Audit (NCAA). This included resuscitation equipment audits $(n=67,91.8 \%)$, participation in the NCCA $(n=66,90.4 \%)$ and audit of Do Not Attempt Cardiopulmonary Resuscitation (DNACPR) documentation ( $n=66,90.4 \%)$.

\section{Basic life support (BLS) and advanced life support (ALS) training provision}

Clinical staff working in the NHS require regular training in resuscitation skills including BLS and ALS. In responding trusts, most delivered only instructor-led BLS training (table $4, \mathrm{n}=51$, $69.9 \%)$. Some trusts $(n=21,28.8 \%)$ reported using other methods such as a combination of instructor-led and self-instruction teaching. Feedback devices were more popular with training, with 51 trusts $(69.9 \%)$ reporting the use of CPR prompt/feedback devices during BLS training and popular devices included 
Table 4 Basic life support and advanced life support training provision

Respondents ( $\mathrm{n}=73$ ) ILCOR recommendation 2015

\begin{tabular}{|c|c|c|}
\hline \multicolumn{3}{|l|}{ BLS training } \\
\hline \multicolumn{3}{|l|}{ Methods of delivery used for BLS teaching, $\mathrm{n}(\%)$} \\
\hline Self-instruction (eg, DVD, e-learning) & $1(1.4)$ & \multirow{3}{*}{$\begin{array}{l}\text { 'We suggest that video and/or computer-based self-instruction with } \\
\text { synchronous or asynchronous hands-on practice may be an effective } \\
\text { alternative to instructor-led courses' (Weak recommendation) }\end{array}$} \\
\hline Instructor-led & $51(69.9)$ & \\
\hline Both & $21(28.8)$ & \\
\hline \multicolumn{3}{|l|}{ CPR prompt/feedback devices used during BLS training, $\mathrm{n}(\%)$} \\
\hline Metronome & $19(26.0)$ & \multirow{4}{*}{$\begin{array}{l}\text { 'We suggest the use of feedback devices that provide directive feedback } \\
\text { on compression rate, depth, release, and hand position during training' } \\
\text { (Weak recommendation) } \\
\text { 'If feedback devices are not available, we suggest the use of tonal } \\
\text { guidance (examples include music or metronome) during training to } \\
\text { improve compression rate only' (Weak recommendation) }\end{array}$} \\
\hline Real-time feedback devices & $35(47.9)$ & \\
\hline Manikin-based feedback & $29(39.8)$ & \\
\hline Prompt/feedback device not used in BLS training & $22(30.1)$ & \\
\hline \multicolumn{3}{|l|}{ Use of high-fidelity manikins during BLS training, $\mathrm{n}(\%)$} \\
\hline Yes & $13(17.8)$ & \multirow{3}{*}{$\begin{array}{l}\text { 'We suggest the use of high-fidelity manikins when training centres/ } \\
\text { organisations have the infrastructure, trained personnel, and resources } \\
\text { to maintain the programme' (Weak recommendation) }\end{array}$} \\
\hline When available & $11(15.1)$ & \\
\hline No & $49(67.1)$ & \\
\hline \multicolumn{3}{|l|}{ ALS training } \\
\hline \multicolumn{3}{|l|}{ CPR prompt/feedback devices used during ALS training, $\mathrm{n}(\%)$} \\
\hline Metronome & $16(21.9)$ & \multirow{4}{*}{$\begin{array}{l}\text { 'We suggest the use of feedback devices that provide directive feedbach } \\
\text { on compression rate, depth, release, and hand position during training' } \\
\text { (Weak recommendation) } \\
\text { 'If feedback devices are not available, we suggest the use of tonal } \\
\text { guidance (examples include music or metronome) during training to } \\
\text { improve compression rate only' (Weak recommendation) }\end{array}$} \\
\hline Real-time feedback devices & $36(49.3)$ & \\
\hline Manikin-based feedback & $33(45.2)$ & \\
\hline Prompt/feedback device not used in ALS training & $12(16.4)$ & \\
\hline \multicolumn{3}{|l|}{ Use of high-fidelity manikins during ALS training, $n(\%)$} \\
\hline Yes routinely & $35(47.9)$ & \multirow{3}{*}{$\begin{array}{l}\text { 'We suggest the use of high-fidelity manikins when training centres/ } \\
\text { organisations have the infrastructure, trained personnel, and resources } \\
\text { to maintain the programme' (Weak recommendation) } \\
\text { 'If high-fidelity manikins are not available, we suggest that the use } \\
\text { of low-fidelity manikins is acceptable for standard ALS training in an } \\
\text { educational setting' (Weak recommendation) }\end{array}$} \\
\hline Used when available & $9(12.3)$ & \\
\hline Not used routinely & $29(39.7)$ & \\
\hline
\end{tabular}

ALS, adult advanced life support; BLS, basic life support; ILCOR, International Liaison Committee on Resuscitation.

real-time feedback devices $(\mathrm{n}=35,47.9 \%)$, manikin-based feedback $(n=29,39.8 \%)$ and metronomes $(n=19,26.0 \%)$. A greater proportion of trusts used CPR prompt/feedback devices during ALS training, with the most commonly used devices being realtime feedback devices $(n=36,49.3 \%)$, manikin-based feedback $(\mathrm{n}=33,45.2 \%)$ and metronome devices $(\mathrm{n}=16,21.9 \%)$.

High-fidelity manikins are rarely used during BLS training by responding trusts (always $\mathrm{n}=13,17.8 \%$; when available $\mathrm{n}=11$, 15.1\%). High-fidelity manikins are used more commonly during ALS training, such that $35(47.9 \%)$ trusts use them regularly during ALS training and 9 (12.3\%) use them when available.

\section{Perceived barriers to implementation of clinical guidelines}

In response to what were the perceived barriers to implementing clinical guidelines, the most frequently reported barriers were financial factors $(n=48,65.8 \%)$ and levels of staffing $(30.1 \%$, $n=22)$. A minority of trusts reported time $(n=5,6.8 \%)$ and the low quality of the evidence underpinning many recommendations $(n=2,2.7 \%)$ as barriers. Eight trusts $(11.0 \%)$ reported no barriers to implementation.

\section{DISCUSSION}

In this survey of the implementation of ILCOR treatment recommendations for in-hospital cardiac arrest at English acute NHS trusts, we found evidence of marked variability in practice. These disparities did not appear to correlate to the strength of treatment recommendation as summarised in tables $2-4$. We found evidence that recommended interventions, such as waveform capnography and ultrasound, are often available only to select patient groups or have yet to be implemented.

The limited compliance in some areas of our survey may be attributable to the short time-span between undertaking our survey and release of the ILCOR treatment recommendations and Resuscitation Council (UK) guidelines. While this was not identified as a barrier to implementation by any trust, it should be recognised that this short duration may not have been sufficient for effective implementation, particularly where additional funding or staffing is required. From this perspective, our survey may provide a useful benchmark to assess implementation rates in the future.

Debriefing provides an interesting example where implementation has not fully reflected the ILCOR treatment recommendation. The implementation of cardiac arrest debriefing is associated with improvements in CPR quality and patient outcome. ${ }^{14-16}$ Effective debriefing is reliant on the availability of objective performance data, such as defibrillator CPR quality downloads or video-recordings. ${ }^{17}$ This is reflected in the ILCOR recommendation that debriefing should be 'datadriven' and 'performance-focused'. ${ }^{10}$ However, our survey found that, while many trusts undertook debriefing, although perhaps on an ad hoc basis, this process was rarely supported by CPR quality data such that the effectiveness of such debriefing may be limited.

To our knowledge, this is the first English survey that has covered a broad range of IHCA practices. Previous surveys of resuscitation have focused on specific areas such as waveform capnography and 
post-resuscitation care. ${ }^{18-21}$ These surveys have similarly identified variability in the implementation of guidelines.

There have been two previous surveys of a broad range of IHCA practices conducted in other countries. ${ }^{22}$ Edelson et al surveyed practice across 439 North American hospitals (response rate 44\%). ${ }^{22}$ The survey reported infrequent implementation in hospitals of capnography (25\%), real-time audiovisual feedback devices (4\%) and debriefing (34\%). The survey by Tirkkonen et al of 51 Finnish hospitals (response rate 93\%) focused on the characteristics of hospital medical emergency and cardiac arrest teams but also reported that, in hospitals with intensive care units, a single hospital (3.4\%) debriefed the team following cardiac arrests and most (72.4\%) used real-time audiovisual feedback technology. ${ }^{23}$ As such, in combination with our survey, these surveys highlight marked variability in in-hospital resuscitation practice both within nations and between nations.

The implementation of evidence-based guidelines in practice is challenging, and there is a need for further work to examine the best approaches to successful implementation in the context of resuscitation. ${ }^{24}$ Our finding that finance and staffing levels were recognised as the key barriers to implementation is unsurprising, particularly in the publicly funded UK National Health Service. However, in contrast to previous surveys, the strength of evidence supporting ILCOR treatment recommendations was not considered a significant barrier to implementation. ${ }^{20} 21$

The key strength of our study is that it is, to our knowledge, the first English survey of in-hospital practices that covers a range of practice areas. It does, however, have some limitations. First, the response rate was 53.3\%. While key demographics (number of beds and hospitals) were broadly similar between responding and non-responding trusts, such a response creates a risk of selection bias. Poor response rates are common in surveys of health professionals, with surveys often reporting response rates similar to that achieved in this study. ${ }^{25}$ We sought to maximise the response rate by limiting the survey length and making multiple contact attempts. Second, we collected data through an electronic survey that was completed by resuscitation officers. Answers were likely based on organisational policy, which may not necessarily reflect actual practice.

\section{Main messages}

- International Liaison Committee on Resuscitation treatment recommendations are yet to be fully implemented across most acute adult NHS hospital trusts

- The key barriers to implementation of treatment recommendations are financial and staffing

- There is variability in resuscitation practice for adult in-hospital cardiac arrest across acute adult NHS hospital trusts

\section{Current research questions}

- How can barriers to implementation of treatment recommendations be addressed?

- What factors influence the prioritisation of implementing some treatment recommendations over others?

- What is the impact of variability in resuscitation practices on patient outcome?

\section{CONCLUSION}

Our survey highlighted variability in IHCA practice across NHS hospitals in England. Many trusts are yet to implement key ILCOR treatment recommendations, with key barriers being financial factors and staffing levels. Our results highlight the need to optimise the final component ('implementation') in the cardiac arrest formula for survival to maximise the likelihood of survival for NHS patients.

Acknowledgements The authors would like to thank all the respondents to the electronic survey for their participation.

Contributors JC, KC and JY designed the study, developed the survey tool, interpreted the data and developed the manuscript for publication. JC collected and analysed the data. JY acts as guarantor for the data.

Funding $\mathrm{KC}$ and JY are supported as NIHR post-doctoral research fellows. Competing interests KC and JY were evidence evaluators for the 2015 ILCOR review process.

Ethics approval The study was approved by the University of Birmingham Ethical Review Committee.

Provenance and peer review Not commissioned; externally peer reviewed. Data sharing statement There are no unpublished data from this study.

Open Access This is an Open Access article distributed in accordance with the terms of the Creative Commons Attribution (CC-BY-NC 4.0) license, which permits others to distribute, remix, adapt and build upon this work, for commercial use, provided the original work is properly cited. See: http://creativecommons.org/ licenses/by/4.0/

(c) Article author(s) (or their employer(s) unless otherwise stated in the text of the article) 2017. All rights reserved. No commercial use is permitted unless otherwise expressly granted.

\section{REFERENCES}

1 Nolan JP, Soar J, Smith GB, et al. Incidence and outcome of in-hospital cardiac arrest in the United Kingdom National ardiac Arrest Audit. Resuscitation 2014;85:987-92.

2 Resuscitation Council (UK). Resuscitation Guidelines. 2015. https://www.resus.org.uk/ resuscitation-guidelines/ (accessed 10 May 2016)

3 Nolan JP, Hazinski MF, Aickin R, et al. Part 1: executive summary: 2015 International Consensus on Cardiopulmonary Resuscitation and emergency cardiovascular care science with treatment recommendations. Resuscitation 2015:95:e1-e31.

4 Hazinski MF, Nolan JP, Aickin R, et al. Part 1: executive summary. 2015 International Consensus on Cardiopulmonary Resuscitation and emergency cardiovascular care science with treatment recommendations. Circulation 2015;132:S2-S39.

5 Morley PT, Lang E, Aickin R, et al. Part 2: evidence evaluation and management of conflicts of interest: 2015 International Consensus on Cardiopulmonary Resuscitation and emergency cardiovascular care science with treatment recommendations. Resuscitation 2015:95:e33-e41.

6 Guyatt GH, Oxman AD, Schünemann HJ, et al. GRADE guidelines: a new series of articles in the Journal of Clinical Epidemiology. J Clin Epidemiol 2011;64:380-2.

7 Søreide E, Morrison L, Hillman K, et al. The formula for survival in resuscitation. Resuscitation 2013:84:1487-93.

8 Nikolaou NI, Welsford M, Beygui F, et al. Part 5: acute coronary syndromes: 2015 International Consensus on Cardiopulmonary Resuscitation and emergency cardiovascular care science with treatment recommendations. Resuscitation 2015;95:e121-46.

9 Perkins GD, Travers AH, Berg RA, et al. Part 3: adult basic life support and automated external defibrillation: 2015 International Consensus on Cardiopulmonary Resuscitation and emergency cardiovascular care science with treatment recommendations. Resuscitation 2015;95:e43-e69.

10 Finn JC, Bhanji F, Lockey A, et al. Part 8: education, implementation, and teams: 2015 International Consensus on Cardiopulmonary Resuscitation and emergency cardiovascular care science with treatment recommendations. Resuscitation 2015:95:e203-24.

11 Soar J, Callaway CW, Aibiki M, et al. Part 4: advanced life support: 2015 International Consensus on Cardiopulmonary Resuscitation and emergency cardiovascular care science with treatment recommendations. Resuscitation 2015;95:e71-120.

12 NHS Choices. Authorities and trust: all NHS trusts. http://www.nhs.uk/ ServiceDirectories/Pages/AcuteTrustListing.aspx (accessed 6 March 2016).

13 Quality standards for cardiopulmonary resuscitation practice and training. 2013. http://www.resus.org.uk/pages/QSCPR_Acute.htm (accessed 8/3/16).

14 Wolfe H, Zebuhr C, Topjian AA, et al. Interdisciplinary ICU cardiac arrest debriefing improves survival outcomes. Crit Care Med 2014;42:1688-95. 
15 Couper K, Kimani PK, Abella BS, et al. The system-wide effect of real-time audiovisual feedback and postevent debriefing for in-hospital cardiac arrest: the cardiopulmonary resuscitation quality improvement initiative. Crit Care Med 2015:43:2321-31.

16 Couper K, Salman B, Soar J, et al. Debriefing to improve outcomes from critical illness: a systematic review and meta-analysis. Intensive Care Med 2013;39:1513-23.

17 Couper K, Perkins GD. Debriefing after resuscitation. Curr Opin Crit Care 2013;19:188-94.

18 Ford AH, Clark T, Reynolds EC, et al. Management of cardiac arrest survivors in UK intensive care units: a survey of practice. J Intensive Care 2016;17:117-21.

19 Turle S, Sherren PB, Nicholson S, et al. Availability and use of capnography for in-hospital cardiac arrests in the United Kingdom. Resuscitation 2015:94:80-4.

20 Binks AC, Murphy RE, Prout RE, et al. Therapeutic hypothermia after cardiac arrest: implementation in UK intensive care units. Anaesthesia 2010;65:260-5.
21 Laver SR, Padkin A, Atalla A, et al. Therapeutic hypothermia after cardiac arrest: a survey of practice in intensive care units in the United Kingdom. Anaesthesia 2006;61:873-7.

22 Edelson DP, Yuen TC, Mancini ME, et al. Hospital cardiac arrest resuscitation practice in the United States: a nationally representative survey. J Hosp Med 2014;9:353-7.

23 Tirkkonen J, Nurmi J, Olkkola KT, et al. Cardiac arrest teams and medical emergency teams in Finland: a nationwide cross-sectional postal survey. Acta Anaesthesiol Scand 2014;58:420-7.

24 Dainty KN, Brooks SC, Morrison LJ. Are the 2010 guidelines on cardiopulmonary resuscitation lost in translation? A call for increased focus on implementation science. Resuscitation 2013;84:422-5.

25 Cook JV, Dickinson HO, Eccles MP. Response rates in postal surveys of healthcare professionals between 1996 and 2005: an observational study. BMC Health Serv Res 2009;9:160. 